\title{
Notas para una investigación futura sobre la historia del internado médico en Colombia, siglo XX. Primera parte: antecedentes europeos y norteamericanos, (siglo XIX e inicios del XX)
}

\author{
Leonardo Palacios-Sánchez'1, María Camila Nuñez-Gómez², Emilio Quevedo-Vélez³
}

\section{RESUMEN}

Este artículo recoge la primera parte de un estudio sobre el desarrollo del internado en la educación médica colombiana. En esta se presenta un panorama histórico-cronológico de los antecedentes europeos y norteamericanos de la aparición y desarrollo de la fiơura del médico interno en la práctica médica. Cubre el periodo comprendido entre el surgimiento del internado durante el gobierno de Napoleón en Francia en el sioglo XIX y su transformación al modelo propuesto por la reforma flexneriana en los Estados Unidos al iniciarse el siglo XX, como punto de partida para la comprensión de dicho proceso en el caso colombiano.

\section{PALABRAS CLAVE}

Educación Médica; Historia; Hospitales; Internado

* Este artículo hace parte de los resultados del proyecto de investigación Historia de la Escuela de Medicina y Ciencias de la Salud de la Universidad del Rosario, 1969-2017, financiado por esta.

1 Profesor Titular de Neurología, Unidad de Neurociencia, Escuela de Medicina y Ciencias de la Salud, Universidad del Rosario, Bogotá, Colombia.

2 Profesional en Artes Liberales en Ciencias Sociales, asistente de investigación del Grupo de Estudios Sociales de las Ciencias, las Tecnologías y las Profesiones de Universidad del Rosario. Estudiante de Maestría en Estudios Sociales de la Ciencia, Universidad Nacional de Colombia.

3 Médico, pediatra, PhD, profesor titular y director del Grupo de Estudios Sociales de las Ciencias, las Tecnologías y las Profesiones, Universidad del Rosario, Bogotá, Colombia. Correspondencia: Leonardo Palacios-Sánchez; leonardo.palacios@urosario.edu.co

Recibido: noviembre 12 de 2017

Aceptado: mayo 07 de 2018

Cómo citar: Palacios-Sánchez L, Nuñez-Gómez MC, Quevedo-Vélez E. Notas para una investigación futura sobre la historia del internado médico en Colombia, siglo XX. Primera parte: antecedentes europeos y norteamericanos, (siglo XIX e inicios del XX). latreia. 2019 Ene-Mar;32(1):33-39. D0I 10.17533/udea.iatreia.v32n1a04. 


\section{SUMMARY}

Notes for a future research on the history of the internship in medicine in Colombia during the 20th century. First part: International background: Europe and North America (19th and 20th centuries)

This article presents the results of the first part of a study about the development of the internship in Colombian medical education. This first part offers a historical-chronological overview of the birth and development of the "internship" in European and North American medical practice, covering the period of time that begins with its appearance during Napoleonian government in France during the $19^{\text {th }}$ century, and its transformation with the model proposed during the flexnerian reform in the United States at the beginning of the $20^{\text {th }}$ century, as a starting point for the understanding of the Colombian case.

\section{KEY WORDS}

History; HospitaIs; Internship and Residency; Medical Education

\section{INTRODUCCIÓN}

En la formación del médico, el último año de la carrera denominado internado rotatorio, es una experiencia muy enriquecedora desde el punto de vista académico y personal, pues le permite afianzar conocimientos y llevar a cabo prácticas bajo supervisión de profesionales, con un mayor grado de autonomía que en los semestres previos. En este artículo se presenta una perspectiva histórica del proceso de conformación $y$ transformación de dicho componente de la carrera de medicina, desde las tendencias que le dieron origen en el panorama internacional.

En efecto, este artículo, basado en la revisión de fuentes secundarias ${ }^{4}$, recoge los resultados de la primera parte de un estudio sobre la aparición y desarrollo de la fiogura del "interno" en la práctica médica y el desarrollo del internado en la educación médica colombiana. Esta primera parte presenta un panorama histórico-cronológico de sus antecedentes, tanto en Europa como en Norteamérica. El análisis se centra en dos procesos: el primero, que se inicia con el surgimiento del internado en Francia durante el gobierno de Napoleón y sus desarrollos durante el siglo XIX; y el segundo, que analiza, por una parte, su implementación un poco más tardía en Norteamérica, durante la segunda mitad del siglo XIX y, por otra, su posterior transformación, puesta en marcha por el modelo propuesto en la reforma flexneriana, en los inicios del siglo XX. Los resultados presentados acá constituyen el punto de partida para la comprensión de dicho proceso en el caso colombiano, el cual será desarrollado en un próximo artículo que recogerá Ios resultados de la segunda parte del estudio, elaborada sobre la revisión de fuentes primarias 5 .

\section{EL INTERNADO EN FRANCIA}

El primer periodo de este proceso de surgimiento del internado, comienza en la Francia post-revolucionaria. No obstante, según la Asociación de Amigos del Antiguo Internado de los Hospitales de París, antes de la aparición del término internado médico o médico interno, desde siglos atrás, en los hospitales de París existía la figura de los médicos compagnons internes (compañeros internos), la cual hacía referencia a los médicos jóvenes, relativamente recién graduados, que servían de apoyo al trabajo de los cirujanos y médicos de los hospitales (1). Algo similar también había ocurrido en Inglaterra durante el siğlo XVIII. Allí, la educación médica se encontraba en una dicotomía teórico-práctica, si alguien quería estudiar medicina, había dos opciones: o acudir a las facultades de medicina de Oxford o Cambridge, costosas y en extremo teóricas, o entrar a un hospital y ser acogido por un médico para tener la apropiada enseñanza práctica, por ejemplo, Edward Jenner, decidiéndose por la segunda opción, fue acogido por su maestro John Hunter como estudiante y como inquilino en su propia casa. Hunter fue "uno de los primeros en tomar como

4 Para el caso de este artículo, entendemos como fuentes secundarias aquellos artículos y libros que se refieren al estudio de cualquier periodo histórico pasado.

5 Entendemos como fuentes primarias, todos aquellos documentos originales producidos durante el periodo que se estudia y que fueron escritos por los actores sociales que participaron en los procesos históricos estudiados. 
inquilinos a algunos alumnos, de ahí lo de "alumno interno" (2). Podemos notar que, desde este entonces, este término era usado también para referirse a estudiantes que vivían o en el hospital o con su maestro, pero no hacían parte de una educación formal de la medicina. Era más bien una distinción de aquellos que vivían en sus propias casas.

Más allá de estos antecedentes eventuales, el término "interno" o "internado" fue realmente oficializado por primera vez en la Escuela de Medicina de Paris, cuando el 10 de febrero de 1802, Napoleón Bonaparte, apoyándose en un documento conocido como eI Informe de los Cinco, instauró el llamado Concurso Republicano para escoger a aquellos estudiantes que tendrían el derecho de realizar una práctica hospitalaria, independiente de los exámenes que se debían aprobar para recibir el título de médico. Quienes ganaban dicha plaza eran llamados médicos internos, para diferenciarlos de aquellos médicos externos que, una vez graduados, se dedicaban a la medicina general. En el contexto del gobierno napoleónico, este concurso tenía como finalidad garantizar un acceso democrático a dicha exclusiva práctica, superando los favoritismos característicos del antiguo régimen. Según su reglamento, el internado podía tener una duración máxima de 4 años (1).

A comienzos del siglo XX, surgieron cambios curriculares en la Facultad de Medicina de París, cuando sus profesores comenzaron a adoptar lo que el historiador español de la medicina, Pedro Laín Entralogo, ha llamado la mentalidad biopatológica, la cual integró los conceptos y prácticas de las mentalidades anatomoclínica, fisiopatológica y etiopatológica ${ }^{6}$, dando origen a la que se llamó la clínica médica francesa clásica. Pero, si bien, esta nueva mentalidad integra los elementos de esas mentalidades anteriores, es decir, la idea de la enfermedad como lesión, como trastorno funcional y como respuesta corporal y orgánica a la agresión externa, ella no es una más en la serie de mentalidades médicas francesas del siglo XIX. Según esta autor, ella "es una rigurosa [...] novedad cualitativa”, que entiende que todos esos procesos patológicos no son aislados sino que hacen parte integral de las funciones vitales del organismo, como una totalidad viviente, "aun cuando algunos de sus conceptos patológicos procediesen de una intuición del ser viviente radicalmente análoga a la suya”, como la mirada integral de la medicina hipocrática y el concepto de la enfermedad como respuesta natural del organismo propuesto por la medicina de Sydenham $(3,4)$.

En ese contexto, el internado se fue convirtiendo en una práctica hospitalaria que, después de recibir el grado, le permitía al médico general quedarse practicando en algún servicio del hospital hasta que sus tutores le certificaran su experiencia para que pudieran dedicarse a la práctica de la especialidad médica a la cual dicho servicio del hospital estuviera dedicado.

Esta práctica, de impronta francesa, fue parcialmente modificada durante el siglo XX por la influencia deI modelo flexneriano y de la medicina norteamericana con su llegada a Europa, durante el periodo de entre guerras $(3,4)$.

\section{EL INTERNADO EN LOS ESTADOS UNIDOS}

En los Estados Unidos de Norteamérica se dio un segundo proceso de desarrollo del internado paralelo al europeo, aunque turo su comienzo un poco más tarde, ya avanzado el siglo XIX. Allí, un médico típico de la década de 1860 recibía su entrenamiento en alguna de las llamadas propietary medical schools. Estas escuelas habían sido creadas como un suplemento al sistema de aprendices médicos, característico de la

\footnotetext{
6 Según Pedro Laín Entralgo, la mentalidad anatomoclínica nació con el texto de Anatomía general (1798) del médico francés Xavier Bichat, y proponía que la enfermedad no era, ni un desequilibrio de los humores, ni un conjunto de síntomas, sino la alteración anatómica (lesión) de los órganos del cuerpo. Dicha mentalidad se consolidó con los trabajos de R. J. Laennec, N. Corvisart y G. L. Bayle, quienes, basados en ese nuevo concepto de enfermedad construyeron una semiología que separaba los síntomas de los signos, al convertir estos últimos en las expresiones físicas de dichas alteraciones anatómicas, las cuales conducen al diagnóstico clínico. La mentalidad fisiopatológica, originada en Alemania en la década de los 40 del siglo XIX, a partir de la comprensión del cuerpo como una máquina termodinámica y de la enfermedad como una alteración energético-material del cuerpo. Dicha mentalidad se consolida en Francia con la aparición del libro Introducción a la Medicina Experimental (1866), de Claude Bernard. La mentalidad etiopatológica es el resultado de la aplicación de los conocimientos aportados por los trabajos de Luis Pasteur y Robert Koch a la clínica médica, a partir de la década de 1870 . Esta mentalidad entiende la enfermedad como el resultado de microorganismos que atacan al cuerpo, el cual se convierte entones en un campo de batalla.
} 
instrucción médica durante el periodo colonial. Pero a partir de la segunda mitad del siglo XIX dichas escuelas habían sustituido este sistema de aprendices médicos por un curso de instrucción que consistía en dos periodos de cuatro meses de clases durante el invierno. El currículo consistía en siete cursos: Anatomía, Fisiología y Patología, Materia médica, Terapéutica y Farmacia, Química y Jurisprudencia médica, Teoría y práctica de la medicina, Principios y práctica de la cirugía, Obstetricia y enfermedades de las mujeres y los niños (5). No obstante, estas escuelas no estaban vinculadas a los hospitales ni a las universidades.

Como una alternativa a esta situación de formación totalmente teórica, con un grupo de escuelas que no garantizaban título, de carácter privado y extramural, que funcionaban durante el periodo de las vacaciones de verano, crearon una instrucción didáctica relacionada con las sesiones regulares de las otras escuelas, consistente en una experiencia clínica que no podía ser obtenida en las escuelas regulares durante las clases de invierno. El énfasis estaba puesto en el examen físico, siguiendo los modelos ya en marcha en Francia (técnicas de percusión, auscultación y palpación) y el uso de instrumentos diagnósticos nuevos como el oftalmoscopio y el otoscopio. Algunas de estas escuelas ofrecían oportunidades para hacer disecciones y obtener experiencia en el vendaje de heridas y en la realización de procedimientos de cirugía menor (5).

Asimismo, para adquirir experiencia práctica el médico joven podía contratarse en un hospital como house pupiI, ya fuera antes o después de recibir el grado. Este tipo de experiencia fue surgiendo entre 1820 y 1830 y se volvió más popular hacia la década de los 50, cuando sus responsabilidades eran más demandantes como resultado del creciente número de cirugías practicadas en los hospitales debido a la introducción de la anestesia (5).

Antes de la Primera Guerra Mundial, ese caroo de house pupil había ido adquiriendo diferentes nombres, dependiendo de las diferentes instituciones que ofrecían dicho puesto de trabajo. Se Ios llamaba entonces "internos", "externos", house pupils, house physicians, residents, resident physicians o house officers, pero en esencia todos eran más o menos iguales, implicaba "una experiencia de uno o dos años para vivir y trabajar en el hospital, atendiendo los asuntos cotidianos de los pacientes y observando los hábitos de práctica de los eminentes médicos de cada momento", el hospital les ofrecía un cuarto donde dormir, alimentación y, a veces, una pequeña remuneración (6). José Félix Patiño confirmaba esta situación cuando decía que "Ios términos 'residente' y 'médico residente' se venían utilizando en los Estados Unidos desde hacía más de un siglo, aun cuando en un principio eran usados indiscriminadamente con el de 'interno'” (7). Como consecuencia de las crecientes exigencias relacionadas con el aumento del número de cirugías en los hospitales entre 1880 y 1890 , el trabajo de los house pupils se fue sobrecargando progresivamente (5).

En 1889, comenzó a aparecer la diferenciación entre ambos cargos. En efecto, en ese año, "el Hospital de Johns Hopkins instituyó el término de 'residente' para identificar al 'joven médico que, habiendo completado el internado continuaba su entrenamiento hospitalario para lograr adiestramiento especial en una rama de la medicina" (7). A partir de entonces, la palabra "interno" quedó relegada a aquellos que hacían una práctica médica hospitalaria paralela a su formación como estudiantes.

Desde 1870, las escuelas médicas ya habían comenzado a vivir una presión para aumentar la duración de sus currículos, con el fin de acomodarse al cada vez más creciente cuerpo de conocimientos médicos. De esta forma, durante el lapso de 30 años, comprendido entre 1870 y 1900 , los tiempos de estudio fueron aumentando desde 16 semanas hasta 32 y, el curso total de la enseñanza médica de 2 a 4 años. Después de la Primera Guerra Mundial, había ya demasiado que enseñar en la carrera de medicina, aún para un currículo de cuatro años. Se hizo necesaria una experiencia que conjugara el conocimiento y la práctica para todos los médicos recién graduados. Asimismo, parecía requerirse un estudio adicional para aquellos que deseaban iniciar una especialidad médica o dedicarse a la investiogación (5).

Debido a la crisis que se estaba presentando en la práctica médica norteamericana (Estados Uniđos-Canadá) como consecuencia del desorden y la falta de rigor en la enseñanza médica, en las décadas finales del sioglo XIX se inició un proceso de cambio que llevaría a una transformación de la educación médica y del internado. Esto comenzó a ocurrir cuando la Asociación Médica Americana recurrió al apoyo de la Fundación Carnegie para el Desarrollo de la Educación, con el 
fin de ayudar a regular la enseñanza médica en los Estados Unidos. En 1908, dicha Fundación contrató a Abraham Flexner, formado en Alemania en psicología educativa, para que realizara un estudio de las condiciones de la enseñanza médica en Norteamérica. Flexner entregó el llamado Informe Flexner en 1910, en el cual, después de presentar un extenso y profunđo análisis de todas las escuelas de medicina en ambos países, proponía el cierre definitivo de todas ellas, resaltando que solo podrían salvarse las que estaban vinculadas a las Universidades, siempre y cuando su estructura curricular y sus métodos de enseñanza se reconstruyeran completamente (8).

Esta propuesta, que ha sido llamada Reforma Flexneriana, consistía en reorganizar la eđucación médica en cuatro años de estudio, siempre y cuando los aspirantes hubiesen completado Ios dos años de College, reglamentarios ya en la educación norteamericana, para poder acceder a cualquier facultad. Durante los años del College el estudiante debería haber adquirido el conocimiento teórico y práctico de las ciencias básicas generales y de las humanidades. Según esta Reforma, los primeros dos años de estudio en la Escuela de Medicina estarían dedicađos al aprendizaje de las ciencias básicas médicas, y los dos años siguientes al estudio de las ciencias clínicas en las salas de los hospitales $(5,8)$.

Frederick T. Gates, asesor del magnate norteamericano del petróleo $y$ creador de la fundación que Ileva su nombre, John D. Rockefeller, se interesó en el estudio del Informe Flexner de 1910 e instó a Rockefeller a apoyar la reforma de la educación médica propuesta en dicho informe. Gates había elaborado para John D. Rockefeller una agenda explícita para la puesta en marcha de las actividades de su Fundación (9). Apoyado en su lectura del Informe Flexner, Gates incluyó, como parte de los cinco puntos de esta agenda, la modernización de la educación médica en los países centrales (Ios Estados Unidos, Gran Bretaña y Francia). Rockefeller se interesó más en el asunto, no solo por las explicaciones de Gates, sino también porque Simon Flexner, hermano de Abraham Flexner, era el director médico del Instituto de Investigaciones Médicas Rockefeller, que el magnate había fundado en 1901.

Con este apoyo de Rockefeller, Gates entró en contacto con Abraham Flexner y después de conversar con él sobre los pormenores de su posible reforma, le ofreció un millón de dólares para su financiamiento, no sin antes preguntarle en cuál universidad ubicarían ese dinero para ponerla en marcha. Flexner les recomendó entonces que la implantación de su reforma se iniciara, como proyecto piloto, en la Universidad de Johns Hopkins, ya que allí trabajaba como decano de la Facultad de Medicina William Welch, patólogo norteamericano formado en Alemania y conocedor de todo el modelo de la universidad de investigación alemana. Rockefeller apoyó esta iniciativa, teniendo en cuenta que también ya había nombrado a Welch en el cargo de presidente en su Instituto de Investigaciones Médicas.

En consecuencia, para apoyar dicha reforma el magnate procedió a hacer la donación para esta Universidad y nombró a Abraham Flexner como director de la Junta de Educación (Rockefeller Education Board) que, a partir de ese momento, dejaría en un segundo plano la educación para niños negros en el sur de Estados Unidos y se centraría en apoyar la reforma de la eđucación médica norteamericana. Una vez implementado el modelo en Johns Hopkins, bajo la tutela del propio WeIch y con el apoyo económico y logístico de la Fundación Rockefeller, las demás facultades de medicina de los Estados Unidos, fueron adoptando paulatinamente el modelo flexneriano (10).

Una vez puesta en marcha la reforma flexneriana, “entre los años de 1910 y 1925, el Comité de Educación Médica de la Asociación Médica Americana, tomó más y más interés en los programas de eđucación graduada, y en 1914, dio a luz la primera lista de Hospitales Aprobados para Internado. Desde 19131915 y [especialmente] en 1919, [dicho Comité] realizó un estudio de los programas hospitalarios que dio como resultado el reconocimiento de que en esa época las facilidades para la educación graduada eran totalmente inadecuadas" (7).

A partir de 1920, y siguiendo las recomendaciones del informe Flexner, en algunas de las escuelas de los Estados Unidos, el foco de la educación médica había comenzado a virar desde el pregrado hacia la educación formal de posgrado, que se recibía después de terminar los cursos teóricos de la escuela médica. Aunque comúnmente los estudiantes de medicina eran considerados como estudiantes de posgrado, técnicamente, el término "estudiante de pregrado" se utilizaba para referirse a aquellos que estaban cursando los cuatro años teóricos y prácticos en la escuela 
de medicina, y la noción de "educación médica graduada" se refería al internado y a la residencia. Ante la discusión de cuál sería la mejor forma de completar la educación médica, algunos de los educadores pensaban que lo más adecuado era agregar un quinto año a la escuela médica (6).

Durante la Primera Guerra Mundial, esta idea tenía muchos defensores, especialmente en las escuelas médicas del medio oeste norteamericano $y$ en una pequeña élite de las escuelas del este. Sin embargo, la mayoría de las escuelas de medicina del país plantearon que no tenían los recursos físicos, financieros ni humanos para aumentar este quinto año. En cambio, la mayoría de los educadores médicos apoyaban la idea de fomentar un periodo de educación posgraduada en los hospitales, lo que ofrecía la ventaja potencial de un trabajo práctico más extenso (6).

De hecho, el entrenamiento para médicos graduados en los hospitales había existido desde tiempo atrás en Ios Estados Unidos, pero estas instituciones solo ofrecían "unos escasos puestos de trabajo asequibles a un pequeño puñado de graduados" (6). "Durante la Primera guerra Mundial, este sistema de puestos hospitalarios fue evolucionando hasta el sistema de internado que fue estandarizado como una experiencia basada en el hospital para completar la eđucación general de los médicos después de recibir su grado en la escuela médica, y la vieja terminología desapareció. Por ejemplo, el Hospital General de Massachusetts reemplazó el termino house pupil por 'interno'”(6).

A partir de ese momento, el Consejo de Educación Médica de la Asociación Médica Americana comenzó a examinar a los hospitales para acreditarlos con el fin de aprobar sus cargos de internado con el mismo rigor con que, dicho consejo, venía evaluando las escuelas médicas. Como resultado de đicha evaluación, en 1919 publicó sus Términos de referencia esenciales para la aprobación de los internados. A partir de 1920, este cambió su nombre por Consejo en Educación Médica y Hospitales. Este proceso convirtió al sistema de práctica hospitalaria en un programa educacional formalmente organizado (6).

Al respecto, el Consejo de Educación Médica y Hospitales observaba que eran necesario "estampar esto con 'educación', no como un trabajo de entrenamiento comparable a aquel de un aprendiz de taller de mecánica” (6). A partir de este cambio, tanto las escuelas médicas como los hospitales apoyaron el crecimiento del internado. Las escuelas comenzaron a considerarlo como una forma adecuada para proveer el entrenamiento adicional de los estudiantes, sin tener que sobrepasar sus capacidades y recursos. Los hospitales, por su parte, vieron a los internos como una solución mayor para su tarea de proveer el cuidado cotidiano de su creciente número de pacientes. Como resultado, hacia 1923, después de la reforma flexneriana, el número de puestos de trabajo para internos en los hospitales fue suficiente para acomodar a todos los graduados de las escuelas médicas (6).

El internado fue entonces ofrecido en tres variedades. El más popular se llamó "internado rotatorio", en el cual los internos rotaban por las diferentes áreas clínicas. En cierto número de hospitales, especialmente aquellos asociados con las escuelas médicas, ofrecían un segundo tipo, llamado "internado rígido", que se realizaba en los servicios de medicina o de cirugía, permaneciendo siempre en uno de los dos. El tercero fue el llamado "internado mixto", que era una mezcla entre el rotatorio y el rígido, el cual permitía una mayor concentración en medicina y cirugía y un tiempo menor en algunas de las especialidades (6).

Estos internados variaban en su longitud temporal, la mayoría de ellos tenían duración de un año, aunque algunos podían durar hasta tres, dependiendo de las políticas propias de los diferentes hospitales. Algunas escuelas comenzaron entonces a exigir la finalización de un internado satisfactorio antes de otorgar el grado de médico. No obstante, a partir de 1939, todas las escuelas de medicina norteamericanas, con excepción de 12 de ellas, comenzaron a conferir el título de médico al final de los cuatro años del currículo, pues consideraban el internado como una fase de posgrado en la educación médica. Después de la Segunda Guerra Mundial, el requerimiento de completar un internado exitoso para recibir el grado de médico en los Estados Unidos desapareció y este entró a formar parte del programa de residencia para las especialidades médicas (6).

\section{EPÍLOGO}

En estas primeras etapas se puede ver cómo el mismo término "interno", tuvo connotaciones diferentes en algunos países de Europa y en los Estados Unidos, durante el siglo XIX y la primera mitad del siglo XX. Es 
interesante observar que, en principio, el internado formó parte de la educación médica de postơrado. Específicamente en Francia, fue el preámbulo de la creación de las especialidades médico-quirúrgicas y, en el caso de los Estados Unidos, a partir de la Primera Guerra Mundial, el internado cambió su ubicación en los planes de estudio médicos para constituirse como el momento final de la formación de pregrado. No obstante, después de la Segunda Guerra Mundial, la actividad del interno volvió a hacer parte de la educación de postơrado. Como veremos en un próximo artículo, en el que se presentará la segunda parte de este estudio, en Colombia se crea y se mantiene como una actividad con la que se remata la formación de pregrado.

Vale la pena remarcar que los procesos de transformación del conocimiento y de la práctica médica están ligados, $y$ en buena parte determinados, por los procesos de reforma curriculares $y$, muy especialmente, aquellos que tienen que ver con la formación práctica en el ámbito hospitalario. Ya desde el siglo XVIII, con las reformas curriculares propuestas por Herman Boerhaave en Leyden, quien incluyó una práctica hospitalaria obligatoria para los estudiantes de medicina, la vinculación de la enseñanza directa a la cabecera del paciente se convirtió en parte esencial de la formación médica.

Fue este ejemplo el que sirvió de orientación para la implementación del internado en Francia durante el consulado Napoleónico, teniendo en cuenta las recomendaciones del informe de los cinco ciudadanos y las revisiones históricas sobre la práctica médica publicadas por Jean-Jacques Cabanis (11). Dicha orientación educativa fue retomada luego por Abraham Flexner, en la primera posguerra del siglo XX, para fundamentar su propuesta de integrar el internado a la formación médica. Tal articulación, ya sea anterior o posterior a la recepción del grado de médico, cambió definitivamente el estilo de trabajo de los profesionales médicos $y$, simultáneamente, impulsó nuevas formas de enseñar la medicina. A través de la historia los cambios en las formas de enseñar medicina han modificado siempre la práctica del médico; a su vez, los cambios de la práctica han incidido en las orientaciones de la formación médica.

\section{CONFLICTOS DE INTERESES}

Ninguno por declarar.

\section{REFERENCIAS BIBLIOGRÁFICAS}

1. Association Amicale des Anciens Internes en Médicine des Hôpitaux de Paris. Histoire de L'internat [Internet]. Paris: AAIHP; 2004 [consultado 5 mars 2018]. Disponible en: http://www.aaihp.fr/Histoire.php

2. Tuells J, Ramírez S. Balmis et Variola. Alicante: Genralitat Valenciana; 2003.

3. Laín Entralgo P. La historia clínica. Historia y teoría del relato patográfico. $2^{\mathrm{a}}$ ed. Barcelona: Salvat; 1961.

4. Laín Entralgo P. El diagnóstico médico. Historia y teoría. Barcelona: Salvat; 1982.

5. Ludmerer KM. Learning to Heal. The Development of American Medical Education. New York: Basic Books; 1985.

6. Ludmerer KM. Time to Heal. American Medical Education from the Turn of the Century to the Era of Managed Care. New York: Ofxord University Press; 1999.

7. Patiño Restrepo JF. La Educación Graduada en Medicina. En: Asociación Colombiana de Facultades de Medicina A, editor. Educación Graduada Programas de acreditación de Hospitales y Certificación de Especialistas. Bogotá: Comité Ejecutivo de ASCOFAME; 1964. p. 9-18.

8. Flexner A. Medical Education in the United States and Canada. A Report to the Carnegie Foundation for the Advancement of Teaching. $2^{\text {nd }}$ ed. New York: The Carnegie Foundation for the Advancement of Teaching; 1910.

9. Solórzano Ramos A. ¿Fiebre dorada o fiebre amariIla? La Fundación Rockefeller en México, 1911-1924. Guadalajara: Universidad de Guadalajara; 1997.

10. Wheatley SC. The Politics of Philanthropy. Abraham Flexner and Medical Education. Madison, Winsconsin: University of Winsconsin Press; 1988.

11. Cabanis PJG. Compendio histórico de las revoluciones y reforma de la medicina. Madrid: Imprenta de Repullés; 1820. 